\title{
Invited Editorial: Does Side Really Matter? Survival Analysis Among Patients with Right- Versus Left-Sided Colon Cancer: A Propensity Score-Adjusted Analysis
}

\author{
Austin J. Livingston, MD' ${ }^{1}$, and Christina E. Bailey, MD, MSCI ${ }^{2}$ \\ ${ }^{1}$ Department of Surgery, Division of Urology, Duke University Medical Center, Durham, NC; ${ }^{2}$ Department of Surgery, \\ Division of Surgical Oncology and Endocrine Surgery, Vanderbilt University Medical Center, Nashville, TN
}

To date, multiple studies have proposed that right-sided colon cancers (proximal to the splenic flexure) and leftsided colon cancers (distal to the splenic flexure) are two distinct diseases. ${ }^{1}$ Colon cancer sidedness has been shown to influence clinical presentation, treatment response, and survival outcomes. ${ }^{2-4}$ In a pooled analysis of six clinical trials, patients with unresectable $R A S$ wild-type metastatic right-sided tumors had worse overall survival (OS), progression free survival, and objective response rates compared with patients with unresectable $R A S$ wild-type metastatic left-sided tumors. Patients with left-sided tumors were also more likely to demonstrate a response to chemotherapy plus estimated glomerular filtration rate (eGFR) antibody therapy. ${ }^{4}$ Similar survival trends have been observed in patients with non-metastatic colon cancer, i.e. patients with right-sided colon cancer have worse OS compared with patients with left-sided colon cancer., Conversely, some studies, all retrospective and populationbased, have found no survival difference based on tumor sidedness, and a few studies have actually demonstrated improved survival in patients with right-sided colon cancer compared with left-sided colon cancer. ${ }^{2,7,8}$

In this issue of Annals of Surgical Oncology, Klose et al. evaluated the 5-year survival for patients with right-versus left-sided stage I-III colon cancer using a prospectively maintained database. ${ }^{9}$ Using a risk-adjusted Cox regression

(C) Society of Surgical Oncology 2021

First Received: 10 December 2020

Accepted: 11 December 2020;

Published Online: 24 September 2021

C. E. Bailey, MD, MSCI

e-mail: christina.e.bailey@vumc.org model and adjusting for confounding baseline patient characteristics by using propensity score-matched analyses, the authors found that tumor sidedness did not have a significant impact on OS, disease-specific survival (DSS), or relative survival (RS). This remained true after accounting for microsatellite instability (MSI) which is associated with improved survival. ${ }^{10}$ On subgroup analysis stratified by stage, there was no difference in OS by stage, but patients with right-sided stage II colon cancer had improved RS compared with patients with left-sided stage II colon cancer. The results of this study add to the ongoing debate of whether patients with right-sided colon cancer have worse prognosis compared with patients with leftsided colon cancer.

As previously mentioned, multiple studies have demonstrated that right- and left-sided colon cancer are distinct entities. Substantial research has gone into identifying differences between cancers based on location, including gene expression, DNA mutations, microbiota, and methylation profile. Right-sided tumors are more commonly associated with MSI, BRAF mutations, and DNA hypermethylation in $\mathrm{CpG}$ islands (termed $\mathrm{CpG}$ island methylator phenotype, or CIMP), whereas left-sided tumors frequently demonstrate chromosomal instability, loss of heterozygosity, and KRAS mutations. ${ }^{11}$

Most studies have demonstrated improved survival for patients with left-sided colon cancer compared with rightsided colon cancer. One of the larger studies was performed by Petrelli et al. This group performed a metaanalysis comparing OS in patients with left-versus rightsided colon cancer. Over 1,400,000 patients were included, and the results demonstrated that patients with left-sided colon cancer had a significant reduction in death independent of cancer stage and receipt of adjuvant chemotherapy 
compared with patients with right-sided colon cancer. ${ }^{3}$ Conversely, the study by Klose et al., demonstrated no change in OS, DSS, or RS for patients with left-versus right-sided colon cancer, and makes the argument that sidedness of colon cancer does not impact survival. ${ }^{9}$ The authors utilized propensity score matching to account for covariates, which differs from other previously published studies. However, several limitations are present that warrant further consideration. For one, this study was unable to account for $B R A F$ mutations, which have previously been shown as a negative prognostic factor and are more routinely found in right-sided cancer. ${ }^{12}$ Furthermore, investigation into the impact of RAS mutation status, HER2 amplification, and DNA hypermethylation were not included. This study also did not include the completeness of mesocolic excision (CME) in their analysis. CME has been shown to be associated with significant disease-free survival benefit in patients with stage I-III colon cancer compared with conventional surgery. ${ }^{13}$ This study did perform additional analysis on tumors with and without MSI. MSI has been shown to be a positive outcome predictor in patients with right-sided colon cancer ${ }^{10}$ and even after excluding MSI tumors, there was still no impact on survival.

Understanding the impact of colon cancer sidedness is complicated when considering the genetic, transcriptomic, proteomic, and microbiologic differences between tumors. These interactions continue to be explored and are important when considering potential drug targets and surgical techniques. Regardless, the difference between right- and left-sided colon cancer is much more complex than can be accounted for simply by tumor location. Outcomes may continue to change as more effective chemotherapy and immunotherapy are developed and our understanding progresses. The mounting evidence shows that right- and leftsided tumors are distinct tumors with varying carcinogenic pathways, presentations, and chemotherapeutic response profiles. However, the data remain unclear when predicting clinical treatment outcomes or patient survival based on subsite for stage I-III tumors. Ultimately, studies such as this current study performed by Klose et al. are important to finding clinically predictive outcome measures that impact patients.

DISCLOSURE Austin J. Livingston and Christina E. Bailey have no potential conflicts of interest to disclose.

\section{REFERENCES}

1. Lee GH, Malietzis G, Askari A, Bernardo D, Al-Hassi HO, Clark SK. Is right-sided colon cancer different to left-sided colorectal cancer? A systematic review. Eur J Surg Oncol. 2015;41(3):300-8. https://doi.org/10.1016/j.ejso.2014.11.001.

2. Karim S, Brennan K, Nanji S, Berry SR, Booth CM. Association between prognosis and tumor laterality in early-stage colon cancer. JAMA Oncol. 2017;3(10):1386-1392. https://doi.org/10. 1001/jamaoncol.2017.1016.

3. Petrelli F, Tomasello G, Borgonovo K, et al. Prognostic survival associated with left-sided vs right-sided colon cancer: a systemic review and meta-analysis. JAMA Oncol. 2017;3(2):211-219. h ttps://doi.org/10.1001/jamaoncol.2016.4227.

4. Arnold D, Lueza B, Douillard JY, et al. Prognostic and predictive value of primary tumour side in patients with RAS wild-type metastatic colorectal cancer treated with chemotherapy and EGFR directed antibodies in six randomized trials. Ann Oncol. 2017;28(8):1713-29. https://doi.org/10.1093/annonc/mdx175.

5. 5. Meguid RA, Slidell MB, Wolfgang CL, et al. Is there a difference in survival between right- versus left-sided colon cancers? Ann Surg Oncol. 2008;15(9):2388-94. https://doi.org/ 10.1245/s10434-008-0015-7.

6. Yahagi M, Okabayashi $\mathrm{K}$, Hasegawa $\mathrm{H}$, et al. The worse prognosis of right-sided compared with left-sided colon cancers: a systematic review and meta-analysis. J Gastrointest Surg. 2016;20(3):648-55. https://doi.org/10.1007/s11605-015-3026-6.

7. Weiss JM, Pfau PR, O'Connor ES, et al. Mortality by stage for right- versus left-sided colon cancer: analysis of surveillance, epidemiology, and end results-medicare data. J Clin Oncol. 2011;29(33):4401-9. https://doi.org/10.1200/jco.2011.36.4414.

8. Warschkow R, Sulz MC, Marti L, et al. Better survival in rightsided versus left-sided stage I-III colon cancer patients. BMC Cancer. 2016;16:554. https://doi.org/10.1186/s12885-016-24120.

9. Klose J, Kloor M, Warschkow R, et al. Does side really matter? Survival analysis among patients with right-versus left-sided colon cancer: a propensity score adjusted analysis. Ann Surg Oncol. 2020. https://doi.org/10.1245/s10434-020-09116-y.

10. Hutchins G, Southward K, Handley L, et al. Value of mismatch repair, KRAS, and BRAF mutations in predicting recurrence and benefits from chemotherapy in colorectal cancer. J Clin Oncol. 2010;29(10):1261-70. https://doi.org/10.1200/jco.2010.30.1366.

11. Baran B, Ozupek NM, Tetik NY, et al. Difference between leftsided and right-sided colorectal cancer: a focused review of literature. Gastroenterology Res. 2018;11(4):264-273. https://doi. org/10.14740/gr1062w.

12. Markowitz SD, Bertagnolli MM. Molecular origins of cancer: Molecular basis of colorectal cancer. N Engl J Med. 2009;361(25):2449-60. https://doi.org/10.1056/nejmra0804588.

13. Bertelsen CA, Neuenschwander AU, Jansen JE, et al. Diseasefree survival after complete mesocolic excision compared with conventional colon cancer surgery: A retrospective, populationbased study. Lancet Oncol. 2015;16(2):161-8. https://doi.org/10. 1016/s1470-2045(14)71168-4.

Publisher's Note Springer Nature remains neutral with regard to jurisdictional claims in published maps and institutional affiliations. 